\title{
Transcatheter aortic valve replacement after mitral valve surgery: Synergistic or incompatible?
}

\author{
Toby Rogers, MD, PhD, a,b and Vinod H. Thourani, MD
}

\footnotetext{
From the Departments of ${ }^{\mathrm{a} C}$ ardiology and ${ }^{\mathrm{c} C}$ ardiac Surgery, MedStar Washington Hospital Center, Washington, DC; and ${ }^{\mathrm{b}}$ Division of Intramural Research, Cardiovascular and Pulmonary Branch, National Heart, Lung, and Blood Institute, National Institutes of Health, Bethesda, Md.

Disclosures: Thourani serves as an advisor and performs research for Edwards Lifesciences. Rogers has nothing to disclose with regard to commercial support.

Received for publication Sept 13, 2017; accepted for publication Sept 15, 2017; available ahead of print Oct 24, 2017.

Address for reprints: Vinod H. Thourani, MD, MedStar Washington Hospital Center, 110 Irving St, NW, Room 6D15G, Washington, DC 20010 (E-mail: vinod.h.thourani@medstar.net).

J Thorac Cardiovasc Surg 2018;155:66-7

0022-5223/\$36.00

Copyright (c) 2017 by The American Association for Thoracic Surgery

https://doi.org/10.1016/j.jtcvs.2017.09.071
}

Advances in transcatheter techniques for structural heart disease offer an opportunity for cardiac surgical patients to avoid the higher morbidity and mortality associated with reoperative surgery should a second pathology develop years after their index procedure. Transcatheter aortic valve replacement (TAVR) has become the standard of care for high-risk patients who have previously undergone coronary artery bypass grafting and later have symptomatic severe aortic stenosis develop. Performing TAVR in patients with a history of mitral valve surgery presents unique challenges, however, because of the anatomic proximity of the valves. All the TAVR pivotal trials excluded patients with surgical prostheses in the mitral, tricuspid, or pulmonary position, and there are therefore very few published data on the safety and effectiveness of TAVR in these patients.

In this issue of the Journal, Squiers and colleagues ${ }^{1}$ present a meta-analysis of case reports and case series of TAVR in patients who had had mitral mechanical or biologic prostheses or annuloplasty rings implanted $13 \pm 6$ years previously. As expected, these were high-risk patients, with a mean Society of Thoracic Surgeons predicted risk of mortality score of $9.9 \%$. Reassuringly, TAVR in this patient population appeared safe, although rates of pacemaker implantation $(20 \%)$ and significant paravalvular leak $(7 \%)$ were higher than for patients with native aortic stenosis undergoing TAVR.

A major concern when performing TAVR in this setting is the potential for mechanical interaction between the transcatheter heart valve (THV) and the existing surgically implanted mitral valve prosthesis. In the setting of a mechanical mitral valve, the TAVR frame can cause one of the mechanical leaflets to "stick" in either the open or closed position. In the case of a bioprosthetic mitral valve, the interaction of the strut can result in misplacement of the THV during implantation or can cause left ventricular outflow obstruction. In the case of a previously repaired mitral valve, the TAVR frame can create an interaction with the anterior leaflet of the mitral valve, leading to late

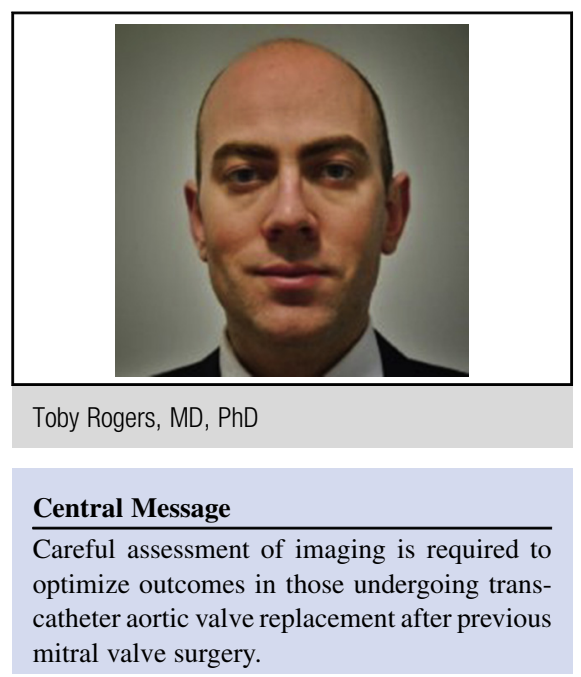

See Article page 63.

leaflet erosion. Fortunately, these complications were rare in this meta-analysis, occurring in only 4 of 137 patients (3\%), although the complication was fatal in 2 patients, required conversion to surgery in 1 , and had to be remedied by snaring of the THV into the ascending aorta and implantation of a second THV in 1.

Some of the included studies predated the routine use of cardiac computed tomography for procedural planning, and data on key anatomic measurements, such as the distance between the aortic annulus and the mitral prosthesis, were missing for $65 \%$ of the patients. If these data were available, it would be very interesting to reanalyze the computed tomography for the 4 patients who had complications to determine whether interaction between the THV and the mitral prosthesis was predictable in any of these cases. Standard 3-dimensional computed tomography is insufficient for this purpose. Instead, contrast-enhanced time-resolved 4-dimensional computed tomography to analyze cardiac motion throughout the cardiac cycle is necessary for true appreciation of the interaction of the aortic anatomy with the mitral valve anatomy, interventricular septum, and left ventricular outflow tract.

Appropriate THV selection is important. Ideal implantation depth for the self-expanding CoreValve THV (Medtronic, Minneapolis, Minn) is $6 \mathrm{~mm}$ or less. The distance of the aortic annulus to the mitral prosthesis was $4.6 \pm 3.3 \mathrm{~mm}$ for mitral bioprostheses in this study, indicating a high likelihood of interaction with the leaflets of 
the mitral prosthesis even if the position of the CoreValve was perfect. In contrast, the balloon-expandable SAPIEN 3 THV (Edwards Lifesciences Corporation, Irvine, Calif) has a height of 15.5 to $22.5 \mathrm{~mm}$ when fully expanded, depending on its nominal diameter. Implantation at a target position of 80:20 relative to the aortic annulus corresponds to an implantation depth of 3.1 to $4.5 \mathrm{~mm}$ and an implantation depth of 1.6 to $2.3 \mathrm{~mm}$ at a target position of $90: 10$. In patients with a short distance between the aortic annulus and the mitral prosthesis, it therefore may well be prudent to select a balloon-expandable valve for the TAVR to reduce the likelihood of mechanical interaction with the mitral prosthesis.

Although prospective studies comparing the performance of various THVs would be welcome, such studies are unlikely to happen. It is therefore important to learn from case series and meta-analyses such as this one. We certainly agree with Squiers and colleagues ${ }^{1}$ that TAVR in patients with a history of mitral valve surgery is associated with increased technical challenges and should only be performed by experienced TAVR implanters. Most recently, we have queried the US Transcatheter Valve Therapies database and have identified 732 cases of TAVR in patients with previous mitral prostheses; the results of this project will be presented in early 2018 and will shed light on this complex patient population. Most important is the recognition that these TAVR procedures require highly individualized procedure planning that uses contrast-enhanced 4-dimensional computed tomographic imaging to maximize technical success and minimize complications.

\section{Reference}

1. Squiers JJ, Potluri S, Brinkman WT, DiMaio JM. Systematic review of transcatheter aortic valve replacement after previous mitral valve surgery. J Thorac Cardiovasc Surg. 2018;155:63-5.e5. 